\title{
Adipöse sterben wirklich vorzeitig
}

\section{In den letzten Jahren erschienen einige zum Teil widersprüchliche Untersuchungen zu der Frage, ob ein erhöhtes Körpergewicht das Sterb- lichkeitsrisiko steigert. Nun wurden lange bekannte Ergebnisse erneut bestätigt: Übergewicht und Adiposi- tas sind lebensbedrohliche Gesund- heitsrisiken.}

\section{Personen mit einem BMI zwischen} 22,5 und $24,9 \mathrm{~kg} / \mathrm{m}^{2}$ hatten das geringste Sterberisiko. Dies traf für Frauen und Männer gleichermaßen zu. Wurden Raucher und Personen mit Krebs bzw. einer Herzerkrankung bei der Auswertung eliminiert, lag die niedrigste Mortalität bei einem BMI zwischen 20 und $25 \mathrm{~kg} / \mathrm{m}^{2}$. Der Zusammenhang zwischen dem BMI und der Mortalität war umso deutlicher, je jünger die Personen waren. Z.B. war das Risiko bei einem BMI von 35-40 $\mathrm{kg} / \mathrm{m}^{2}$ bei den $20-49$-Jährigen um $44 \%$ höher als bei den 70-84-Jährigen.

In Studien mit einem längeren Verlauf zeigte sich ein höheres adipositasbedingtes Sterblichkeitsrisiko, vor allem in den höheren $\mathrm{Ge}$ wichtsklassen. Bei Studien mit einer Beobachtungsdauer von mehr als 15 Jahren im BMI-Bereich 40-49,9 kg/m ${ }^{2}$ war das Risiko im Ver- gleich zum Referenzbereich (BMI 22,5$24,9 \mathrm{~kg} / \mathrm{m}^{2}$ ) um das 3,1-Fache erhöht, bei solchen unter fünf Jahren nur um das 2,3-Fache.

\section{Kommentar}

Seit der Veröffentlichung einer Studie der amerikanischen Lebensversicherungsgesellschaft Metropolitan Life Insurance Company im Jahr 1959 hat sich an der Sicht, ob und wie das Körpergewicht ein Risiko für eine erhöhte Mortalität darstellt, wenig geändert. In den Auswertungen von großen Kollektiven wie der Nurses Health Study (1995), der Cancer Prevention Study (1999), der Framingham Heart Study (2003), dem National Institute of Health Survey (2006), der EPICStudie (2008) und den Prospective Studies Colloboration (2009) zeigten sich ähnliche Ergebnisse wie in der vorliegenden Studie. Der BMI mit der geringsten Mortalität lag in einem Bereich zwischen 20 und $27 \mathrm{~kg} / \mathrm{m}^{2}$.

A. WIRTH =

- A. P. Berrington de Gonzalez et al. Body-mass index and mortality among 1.46 million white adults. N. Engl. J. Med. 363 (2010) 2211-2219

\section{Kraniosakrale Therapie bei Zerebralparese}

\begin{abstract}
Anhänger der kraniosakralen Therapie (eine Variante der Osteopathie) gehen davon aus, dass es ein Pulssystem der Gehirn- und Rückenmarksflüssigkeit gibt. Diesen Puls wollen die Therapeuten ertasten und durch gezielte Manipulationen stärken und harmonisieren.
\end{abstract}

- Britische Pädiater haben 142 Kinder mit Zerebralparese randomisiert. Die Experimentalgruppe erhielt über ein halbes Jahr regelmäßig kraniosakrale
Therapie, während die Kontrollgruppe unbehandelt blieb. Bei Therapieende zeigten sich keine signifikanten Gruppenunterschiede bezüglich Funktion oder Lebensqualität.

\section{Kommentar}

Wie so viele alternative Methoden, ist die kraniosakrale Therapie beliebt, wenig plausibel und kaum untersucht. Die vorliegende Studie ist gut gemacht und eine der ersten Untersuchungen in diesem Bereich. Die Ergebnisse sind überzeugend negativ. Es wäre faszinierend zu sehen, ob jetzt die Beliebtheit der kraniosakralen Therapie abnimmt. Ich fürchte jedoch, dass auf diesem Gebiet Evidenz keinen hohen Stellenwert hat und von quasi religiösem Glauben in den Schatten gestellt wird.

E. ERNST =

\footnotetext{
- K. Wyatt et al.

Cranial osteopathy for children with cerebral palsy: a randomised controlled trial. Arch. Dis. Child. doi:10.1136/adc.2010.199877 Published Online First 24 February 2011
} 